\title{
On the Topographical Effect upon the Perturbations of the Middle Latitude Westerlies
}

\author{
by \\ M. Magata \\ Meteorological Research Institute \\ (Received April 8, 1957)
}

\begin{abstract}
In order to discuss the general character of the perturbations of the westerlies produced by the mountains such as the Himalayas and the Rockies, we solve the stationary vorticity equation for the equivalent-barotropic level, taking into account topographical factors. It is shown that owing to the intensity of the general current, there are critical cases in which a single jet stream or two branches of it appear and these can be regarded as characterizing the normal state at the $500-\mathrm{mb}$ level in January or June respectively. Especially the persistence of a ridge over the Sea of Okhotsk in the Baiu season, is thought to be produced in connection with the Himalayas. The effect of shear of the general current and friction upon the flow pattern are also discussed.
\end{abstract}

\section{Introduction}

The main problems in the dynamical effect of the mountains upon the perturbations of the westerlies have been given solutions by many authors, such as Charney and Eliassen (1949), Bolin (1950), Masuda (1955), Gambo (1956) and Murakami (1956). They showed that when the general current is intense as in January, a main trough is produced and a marked maximum of wind speed as the jet stream appears in the lee of the mountain. In June, however, we find two branches of the jet stream over the Far East, as pointed out by Murakami (1951). Murakami guessed from the computed flow pattern for June that there is a tendency to split in the jet stream associated with the Himalayas. But whether this splitting of the jet stream over the Far East is mainly caused by the effect of the Himalayas, has not been settled. It is the purpose of the present article to make clear this point. In order to simplify the numerical treatment we use the virtual mountain of a simple shape in place of the real mountain and inquire into how the flow pattern varies, as the intensity of the general current varies, by solving the stationary vorticity equation for the equivalentbarotropic level, taking into account topographical factors. Consequently we knew that owing to the intensity of the general current, there were critical cases in which a single jet stream or two branches of a jet stream appeared and these could be regarded as characterizing the normal state at the $500-\mathrm{mb}$ level in January or June respectively. Especially when the two branches of jet stream 
appear, a pronounced ridge is produced north-east of the mountain and this ridge is thought to be associated with the formation of the Okhotsk Sea high in the Baiu season. Namely, the Okhotsk Sea high is thought to be produced by the dynamical effect of the Himalayas.

\section{The case of a uniform general current without friction}

We start from the following equation for the equivalent-barotropic level introduced by CHARNEY and ELIASSEN,

$$
\begin{gathered}
\left(\frac{\partial}{\partial t}+u \frac{\partial}{\partial x}+v \frac{\partial}{\partial y}+\kappa F\right) \nabla^{2} z+\beta \frac{\partial z}{\partial x}-\lambda^{2} \frac{\partial z}{\partial t}=-\kappa \lambda^{2}\left(u \frac{\partial h}{\partial x}+v \frac{\partial h}{\partial y}\right), \\
u=-\frac{g}{f} \frac{\partial z}{\partial y}, \quad v=\frac{g}{f} \frac{\partial z}{\partial x}, \quad \beta=\frac{d f}{d y}, \quad \lambda^{2}=\frac{f^{2}}{g H},
\end{gathered}
$$

in which $z$ is the height of isobaric surface, $f$ is the Coriolis parameter, $h$ is the height of the ground, $g$ is the acceleration of gravity, $F$ is the constant associated with friction, and we assume that the velocity $u_{0}, v_{0}$ and vorticity $\zeta_{0}$ near the ground as a certain fraction $\kappa$ of the velocity $u, v$ and vorticity $\zeta$ at the equivalentbarotropic level, i.e.

$$
u_{0}=\kappa u, \quad v_{0}=\kappa v, \quad \zeta_{0}=\kappa \zeta .
$$

If the motion is stationary and frictionless, $\frac{\partial z}{\partial t}=0, F=0$, thus substituting (2) into (1)

where

$$
\frac{g}{f} J\left(\nabla^{2} z+\frac{f^{2}}{g}+\kappa \lambda^{2} h, z\right)=0,
$$

$$
J(A, B)=\frac{\partial A}{\partial x} \frac{\partial B}{\partial y}-\frac{\partial A}{\partial y} \frac{\partial B}{\partial x},
$$

so that assuming $G(z)$ an arbitrary function,

$$
\nabla^{2} z+\frac{f^{2}}{g}+\kappa \lambda^{2} h={ }_{g}^{f} G(z)
$$

We expand $z$ as follows

$$
z=z_{0}+\kappa z_{1}+\kappa^{2} z_{2}+\cdots \cdots+\kappa^{n} z_{n}+\cdots \cdots \cdot
$$

Substituting this power series of $\kappa$ in (3) and equating coefficients of different powers of $\kappa$, the differential equations for $z_{n}(n=0,1,2, \cdots \cdots)$ become

$$
\begin{aligned}
& \nabla^{2} z_{0}+\frac{f^{2}}{g}=\frac{f}{g} G\left(z_{0}\right), \\
& \nabla^{2} z_{1}-\frac{f}{g} G^{\prime}\left(z_{0}\right) z_{1}=-\lambda^{2} h, \\
& \nabla^{2} z_{2}-\frac{f}{g} G^{\prime}\left(z_{0}\right) z_{2}=\frac{1}{2} \frac{f}{g} G^{\prime \prime}\left(z_{0}\right) z_{1}^{2},
\end{aligned}
$$


(7)

$$
\nabla^{2} z_{3}-{ }_{g}^{f} G^{\prime}\left(z_{0}\right) z_{3}=\frac{f}{g} G^{\prime \prime}\left(z_{0}\right) z_{1} z_{2}+\frac{1}{6} \frac{f}{g} G^{\prime \prime \prime}\left(z_{0}\right) z_{1}^{3},
$$

Though (5), (6), (7) $\cdots \cdots$ are linear equations on $z_{1}, z_{2}, z_{3} \cdots \cdots$ respectively, we must decide an arbitrary function $G(z)$. If $z_{0}$ is the height of the isobaric surface corresponding to the general current $U(y)$, we have

$$
\frac{\partial z_{0}}{\partial y}=-\frac{f}{g} U(y) \text {. }
$$

Substituting (8) in (4),

$$
G\left(z_{0}\right)=\frac{f}{g}\left(f-\frac{d U}{d y}\right)
$$

Thus, assuming $\frac{f}{g}$ to be constant,

$$
\begin{gathered}
G^{\prime}\left(z_{0}\right)=\frac{d G}{d y} / \frac{d z_{0}}{d y}=-\frac{\beta-U^{\prime \prime}}{U}, \\
G^{\prime \prime}\left(z_{0}\right)=\frac{d G^{\prime}}{d y} / \frac{d z_{0}}{d y}=\frac{g\left(U^{\prime} U^{\prime \prime}-U U^{\prime \prime \prime}-\beta U^{\prime}\right)}{f U^{3}} .
\end{gathered}
$$

Substituting (10) and (11) in (5) and (6), we get,

$$
\begin{aligned}
\nabla^{2} z_{1}+\frac{\beta-U^{\prime \prime}}{U} z_{1} & =-\lambda^{2} h, \\
\nabla^{2} z_{2}+\frac{\beta-U^{\prime \prime}}{U} z_{2} & =\frac{1}{2} \frac{g\left(U^{\prime} U^{\prime \prime}-U U^{\prime \prime \prime}-\beta U^{\prime}\right)}{f U^{3}} z_{1}{ }^{2} .
\end{aligned}
$$

Now, assuming the general current to be uniform, let

$$
U(y)=U, \quad \text { (const.) }
$$

then (12) and (13) become

$$
\begin{aligned}
& \nabla^{2} z_{1}+\frac{\beta}{U} z_{1}=-\lambda^{2} h, \\
& \nabla^{2} z_{2}+\frac{\beta}{U} z_{2}=0 .
\end{aligned}
$$

In the same way,

$$
\nabla^{2} z_{n}+\frac{\beta}{U} z_{n}=0 . \quad(n=3,4,5, \cdots \cdots)
$$

Thus we may put $z_{n}(n=2,3, \cdots \cdots)$ to be zero, so that

$$
z=z_{0}+\kappa z_{1} \text {. }
$$

The height contour of the mountain is expanded as follows :

$$
-\lambda^{2} h=\sum_{n=1}^{\infty} F_{n}(x) \sin \frac{n \pi}{a} y,
$$


where

$$
F_{n}(x)=-\frac{2 \lambda^{2}}{a} \int_{0}^{a} h(x, y) \sin \frac{n \pi}{a} \cdot y d y .
$$

Similarly we expand $z_{1}$ as follows :

$$
z_{1}=\sum_{n=1}^{\infty} \phi_{n}(x) \sin \frac{n \pi}{a} y .
$$

Substituting (16) and (17) in (14), we get the differential equation for $\phi_{n}(x)$

$$
\frac{d^{2} \phi_{n}}{d x^{2}}+\left(\frac{\beta}{U}-\frac{n^{2} \pi^{2}}{a^{2}}\right) \phi_{n}=F_{n}(x) . \quad(n=1,2,3, \cdots \cdots)
$$

We assume that $h$, so that $F_{n}(x)$ not zero only in the finite area and the boundary condition at $x= \pm \infty$ is as follows:

$$
\lim _{x \rightarrow-\infty} \phi_{n}=0, \quad \lim _{x \rightarrow+\infty} \psi_{n}=\text { finite. }
$$

We can get the solutions of the equation (18) satisfying the boundary condition (19) as follows :

i) When $\frac{\beta}{U}>\frac{n^{2} \pi^{2}}{a^{2}} \quad\left(\sigma_{n}=\sqrt{\frac{\beta}{U}-\frac{n^{2} \pi^{2}}{a^{2}}}\right)$

$$
\phi_{n}=\frac{1}{\sigma_{n}} \int_{-\infty}^{x} F_{n}(\xi) \sin \sigma_{n}(x-\xi) d \xi .
$$

ii) When $\frac{\beta}{U}<\frac{n^{2} \pi^{2}}{a^{2}} \quad\left(\sigma_{n}=\sqrt{\frac{n^{2} \pi^{2}}{a^{2}}-\frac{\beta}{U}}\right)$

$$
\psi_{n}=\frac{1}{\sigma_{n}} \int_{-\infty}^{x} F_{n}(\xi) \sinh \sigma_{n}(x-\xi) d \xi-\frac{e^{\sigma_{n} x}}{2 \sigma_{n}} \int_{-\infty}^{\infty} F_{n}(\xi) e^{-\sigma_{n} \xi} d \xi
$$

iii) When $\frac{\beta}{U}=\frac{n^{2} \pi^{2}}{a^{2}}$, there is no solution satisfying the boundary condition (19). But the solution of (18) which satisfies the boundary condition $\lim _{x \rightarrow-\infty} \phi_{n}=0, \quad \lim _{x \rightarrow+\infty} \phi_{n}=\infty$ becomes as follows :

$$
\psi_{n}=\int_{-\infty}^{x} F_{n}(\xi)(x-\xi) d \xi
$$

The first several terms of (17) to be $\frac{\beta}{U}>\frac{n^{2} \pi^{2}}{a^{2}}$ i.e. $n<\frac{a}{\pi} \sqrt{\frac{\beta}{U}}$, are waveshaped, as may be seen from (20), but the remaining terms are exponentially damping. As will be afterwards shown, the main character of $z$ is decided by the first several terms to be waveshaped. Fig. 1 shows the wave length $L_{n}=\frac{2 \pi}{\sigma_{n}}$ of $\psi_{n}(n=1,2,3,4,5)$ in (20)

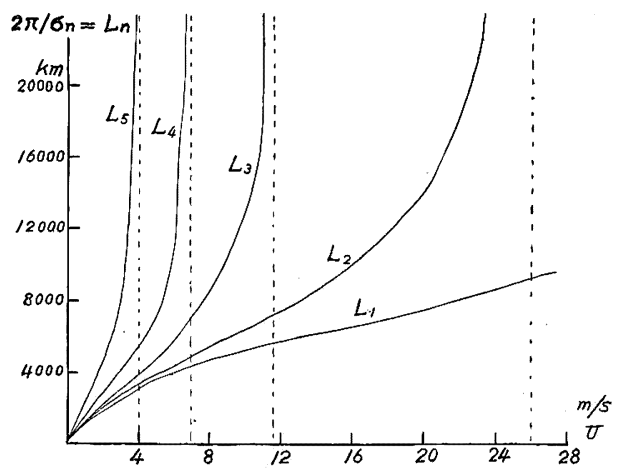

Fig. 1. The wave lengths of the perturbations produced by the mountain in the case of uniform general current without friction. 
for varying values of $U$. Values of $U$ to be

$$
\frac{\beta}{U}=\frac{n^{2} \pi^{2}}{a^{2}},
$$

become as follows:

\begin{tabular}{|c|c|c|c|c|c|}
\hline $\begin{array}{l} \\
a \quad n\end{array}$ & 1 & 2 & 3 & 4 & 5 \\
\hline $6,600 \mathrm{~km}$ & $71.6 \mathrm{~m} / \mathrm{s}$ & 17.9 & 8.0 & 4.5 & 2.9 \\
\hline $8,000 \mathrm{~km}$ & 105.0 & 26.0 & 11.6 & 6.5 & 4.2 \\
\hline
\end{tabular}

We will designate these critical values of $U$.

As may be seen from Fig. 1, if values of $U$ cross a critical value, the number of $\phi_{n}$ wave-shaped will reduce, that is, when $U>26 \mathrm{~m} / \mathrm{s}$, the first term of (17) is wave-shaped as (20) and the remaining terms are exponentially damping as (21), and when $26 \mathrm{~m} / \mathrm{s}>U>11.6 \mathrm{~m} / \mathrm{s}$ the first two terms of (17) are wave-shaped and the remaining are damping, and so on. Next, the distribution of $z$ will be computed, by giving the virtual shape of the mountain as the follows:

$$
\begin{array}{ll}
h=\kappa(y)\left(1+\cos \frac{\pi}{\alpha} x\right) & (|x| \leqq \alpha) \\
=0, & (|x|>\alpha)
\end{array}
$$

where $\kappa(y)$ is a function of $y$ only and $\alpha$ is a constant expressing the width of mountain. Let $\kappa(y)$ be expanded as follows :

$$
-\lambda^{2} \kappa(y)=\sum_{n=1}^{\infty} A_{n} \sin \underset{a}{n \pi} y
$$

then $F_{n}(x)$ of (18) becomes

$$
\begin{aligned}
F_{n}(x) & =A_{n}\left(1+\cos \frac{\pi}{\alpha} x\right) & & (|x| \leqq \alpha) \\
& =0 . & & (|x|>\alpha)
\end{aligned}
$$

In this case (20), (21) and (22) become as follows:

i) When $\frac{\beta}{U}>\frac{n^{2} \pi^{2}}{a^{2}}$

$$
\begin{array}{rlrl}
\phi_{n} & =0 & & (x<-\alpha) \\
& =A_{n}\left\{\frac{1}{\sigma_{n}^{2}}-\frac{\alpha^{2}}{\pi^{2}-\alpha^{2} \sigma_{n}{ }^{2}} \cos \frac{\pi}{\alpha} x-\frac{\pi^{2}}{\sigma_{n}^{2}\left(\pi^{2}-\alpha^{2} \sigma_{n}^{2}\right)} \cos \sigma_{n}(x+\alpha)\right\} & (|x| \leqq \alpha) \\
& =A_{n} \frac{2 \pi^{2} \sin \sigma_{n} \alpha}{\sigma_{n}{ }^{2}\left(\pi^{2}-\alpha^{2} \sigma_{n}{ }^{2}\right)} \sin \sigma_{n} x, & & (x>\alpha)
\end{array}
$$

where

$$
\sigma_{n}^{2}=\frac{\beta}{U}-\frac{n^{2} \pi^{2}}{a^{2}} .
$$

ii) When $\frac{\beta}{U}<\frac{n^{2} \pi^{2}}{a^{2}}$

$$
\phi_{n}=-A_{n} \frac{\pi^{2} \sinh \sigma_{n} \alpha}{\sigma_{n}{ }^{2}\left(\pi^{2}+\alpha^{2} \sigma_{n}{ }^{2}\right)} e^{\sigma_{n} x}
$$




$$
\begin{array}{lrl}
=A_{n}\left(-\frac{1}{\sigma_{n}{ }^{2}}+\frac{\pi^{2} e^{-\sigma_{n} \alpha}}{\sigma_{n}{ }^{2}\left(\pi^{2}+\alpha^{2} \sigma_{n}{ }^{2}\right)} \cosh \sigma_{n} x-\frac{\alpha^{2}}{\left.\pi^{2}+\alpha^{2}{\sigma_{n}{ }^{2}} \cos \frac{\pi}{\alpha} x\right)}\right. & (|x| \leqq \alpha) \\
=-A_{n} \frac{\pi^{2} \sinh \sigma_{n} \alpha}{\sigma_{n}{ }^{2}\left(\pi^{2}+\alpha^{2} \sigma_{n}{ }^{2}\right)} e^{-\sigma_{n} x}, & (x>\alpha)
\end{array}
$$

where

$$
\sigma_{n}^{2}=\frac{n^{2} \pi^{2}}{a^{2}}-\frac{\beta}{U}
$$

iii) When $\frac{\beta}{U}=\frac{n^{2} \pi^{2}}{a^{2}}$

$$
\begin{aligned}
\phi_{n} & =0 \\
& =A_{n}\left(\frac{x^{2}}{2}+\alpha x+\frac{\alpha^{2}}{2}-\frac{\alpha^{2}}{\pi^{2}}-\frac{\alpha^{2}}{\pi^{2}} \cos \frac{\pi}{\alpha} x\right) \\
& =2 \alpha A_{n} x .
\end{aligned}
$$

$$
\begin{array}{r}
(x<-\alpha) \\
(|x| \leqq \alpha) \\
(x>\alpha)
\end{array}
$$

We put $\kappa(y)$ of (25) as follows:

that is

$$
\begin{array}{rlrl}
\kappa(y) & =\frac{h_{0}}{4}\left(1+\cos \frac{\pi}{\alpha}(y-c)\right) & & (y-c \mid \leqq \alpha) \\
& =0 & & (|y-c|>\alpha), \\
h=\frac{h_{0}}{4}\left(1+\cos \frac{\pi}{\alpha}(y-c)\right)\left(1+\cos \frac{\pi}{\alpha} x\right) & & \left(\begin{array}{l}
|x| \leqq \alpha \\
|y-c| \leqq \alpha
\end{array}\right) .
\end{array}
$$

Fig. 3 shows the distributions of $z$ for the different values of $U$ where $\alpha=2,000 \mathrm{~km}, \varepsilon \frac{h_{0}}{H}=\frac{1}{10}$ and $a=8,000 \mathrm{~km}$. In order to look into the rate of convergence of infinite series (17), the functions $\phi_{1}, \phi_{2}, \phi_{3}, \phi_{4}$ and $\phi_{5}$ are shown when $U=10 \mathrm{~m} / \mathrm{s}$ in Fig. 2. As may be seen from this figure, the main character of the distribution is found to be decided by the first several wave-shaped terms of (17).

Now the normal height distributions of $500 \mathrm{mb}$-level in the lee of the Himalayas are shown in Fig. 4. We will compare these with the computed height distributions in Fig. 3. Fig. 3 (a) shows height distribution in the critical case when $U=26 \mathrm{~m} / \mathrm{s}$. The main character in this case is the appearance of a remarkable jet stream in the lee of the mountain

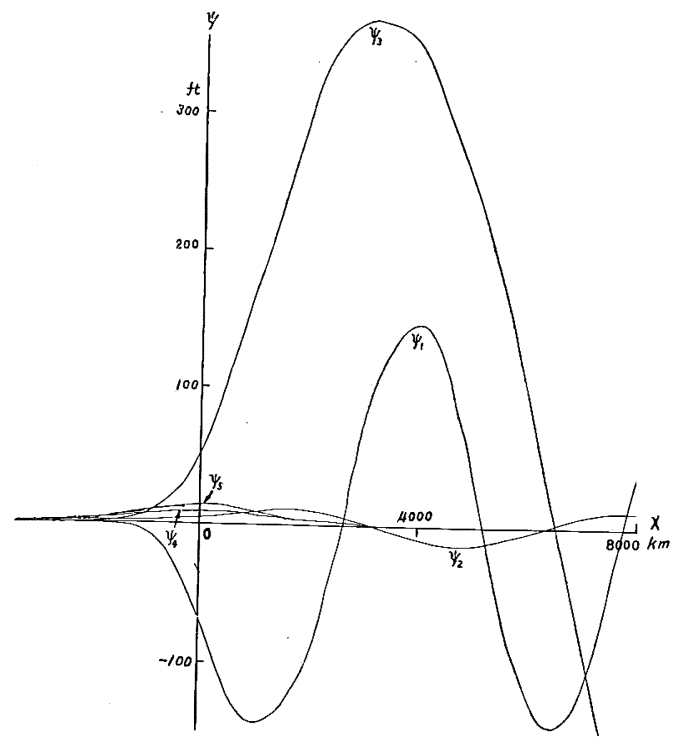

Fig. 2. The profiles of $\phi_{1}(x), \phi_{2}(x), \phi_{3}(x), \phi_{4}(x)$ and $\phi_{5}(x)$ in the series $z_{1}=\sum_{n=1}^{\infty} \phi_{n}(x) \sin \frac{n \pi}{a} y$ when $U=10 \mathrm{~m} / \mathrm{s}$. which is similar to the normal height distribution in January (Fig. $4(\mathrm{a})$ ). But there is serious discrepancy in the strength of the general currents. We will later discuss this point. Fig. 3 (b) shows the 


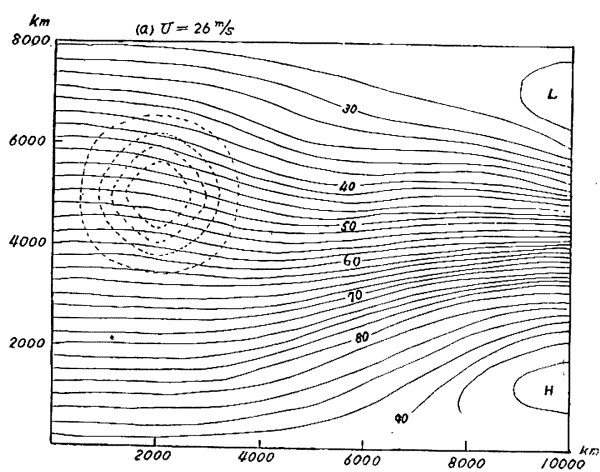

(a)

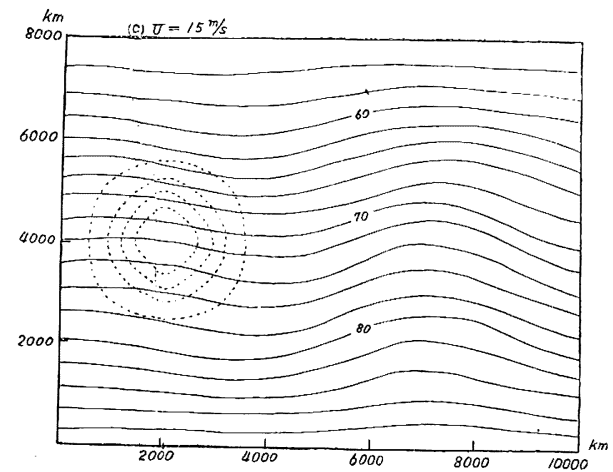

(c)

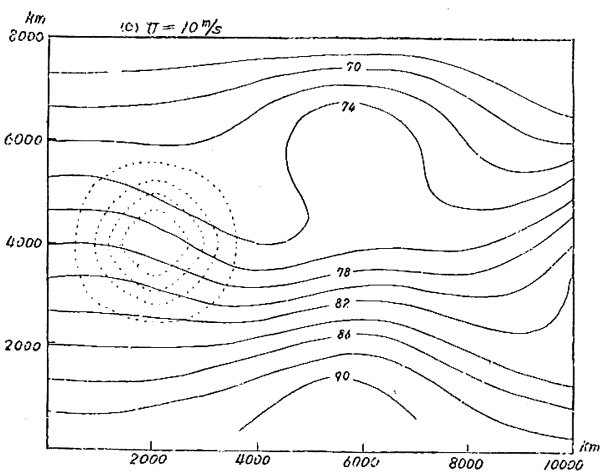

(e)

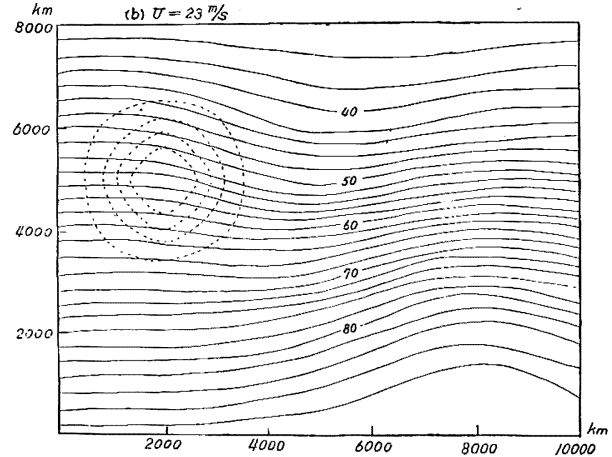

(b)

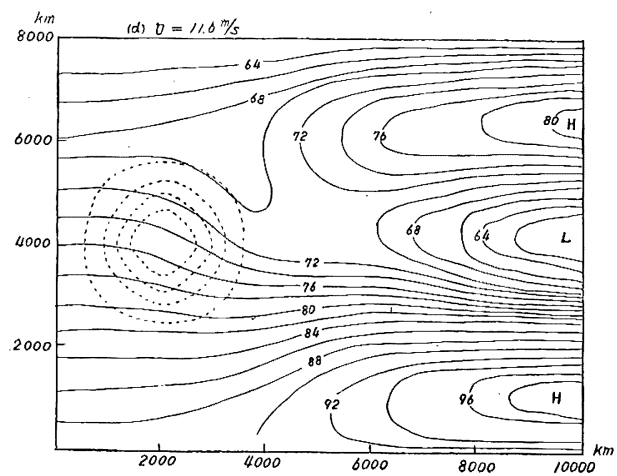

(d)

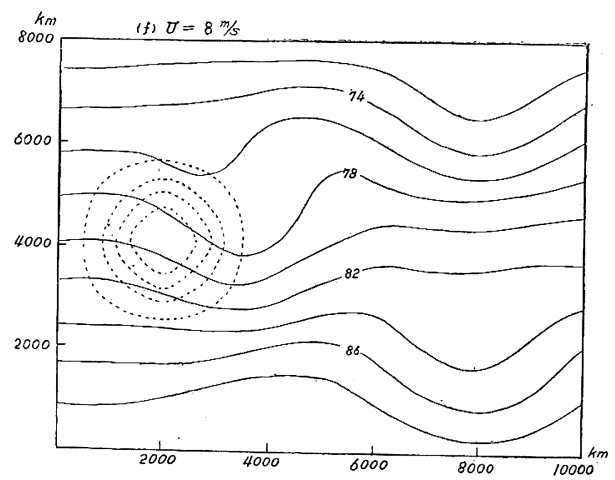

( f )

Fig. 3. Computed height contours at 200 feet intervals, in the cases of

(a) $U=26 \mathrm{~m} / \mathrm{s}$ (critical value), (b) $U=23 \mathrm{~m} / \mathrm{s}$, (c) $U=15 \mathrm{~m} / \mathrm{s}$,

(d) $U=11.6 \mathrm{~m} / \mathrm{s}$ (critical value), (e) $U=10 \mathrm{~m} / \mathrm{s}$ and (f) $U=8 \mathrm{~m} / \mathrm{s}$.

Dashed lines show contours of the mountain. 


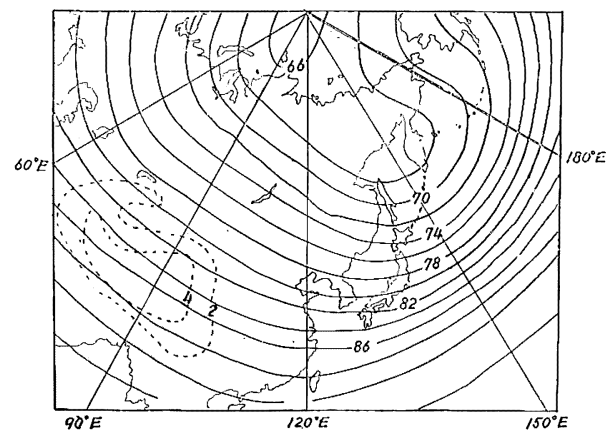

(a)

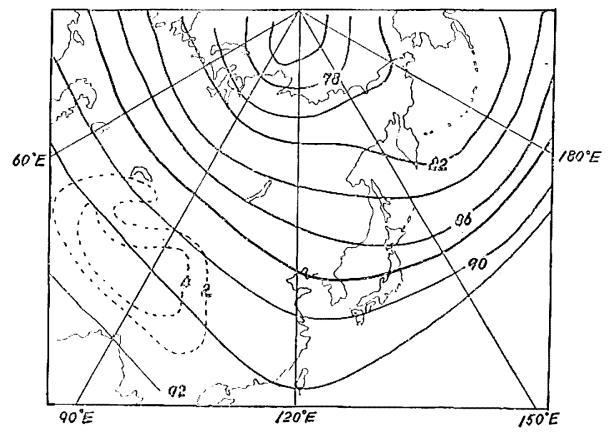

(b)

Fig. 4. Normal height contours on $500 \mathrm{mb}$ level for January (a) and June (b). Dashed line show height contours of the Himalayas. (Unit in $\mathrm{km}$ ).

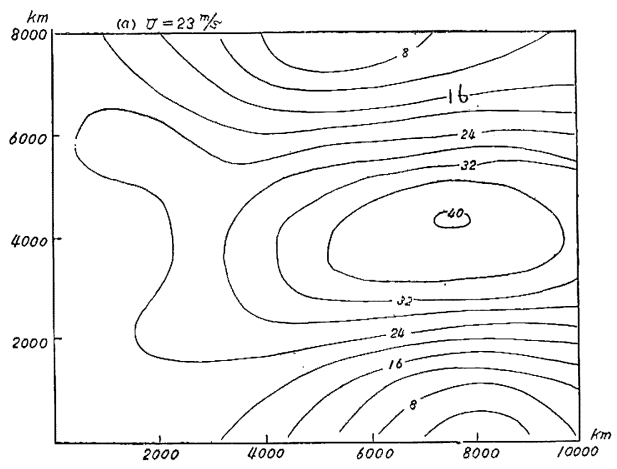

(a)

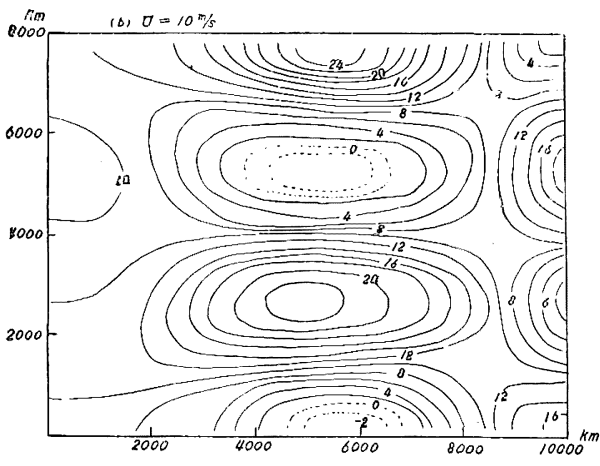

(b)

Fig. 5. Computed distributions of geostrophic zonal wind velocity $(\mathrm{m} / \mathrm{s})$ when (a) $U=23 \mathrm{~m} / \mathrm{s}$ and (b) $U=10 \mathrm{~m} / \mathrm{s}$. Isolines are drawn at $4 \mathrm{~m} / \mathrm{s}$ intervals in (a) and at $2 \mathrm{~m} / \mathrm{s}$ intervals in (b).

case when $U=23 \mathrm{~m} / \mathrm{s}$ and a somewhat weak jet stream appears. Fig. $3(\mathrm{c})$ shows the height distribution when $U=15 \mathrm{~m} / \mathrm{s}$ and no jet stream appears and a marked trough and ridge are produced. Fig. 5(a) shows the distribution of the zonal wind velocity when $U=23 \mathrm{~m} / \mathrm{s}$. Fig. $3(\mathrm{~d})$ shows the height distribution in the critical case when $U=11.6 \mathrm{~m} / \mathrm{s}$. The remarkable features in this case are the appearance of two branches of jet stream and a marked high pressure to the north-east of the mountain. Fig. 3(e) shows the height distribution for $U=10 \mathrm{~m} / \mathrm{s}$ which is qualitatively similar to the normal height distribution in June (Fig. 4(b)). According to Murakamis study, in the Baiu season there is a remarkable splitting of the jet stream at upper levels in the vicinity of the Japan Islands and it is well known that there is anticyclongenesis over the Sea of Okhotsk in the Baiu season. OKuTA confirmed in 1950 that the Okhotsk Sea high was of the warm type extending to high levels contrary to the common notion of cold anticyclone. Fig. 5(b) shows the distribution of the zonal wind velocity when $U=10 \mathrm{~m} / \mathrm{s}$. In this figure there is a remarkable splitting of the jet stream. The marked ridge to the northeast of the mountain in Fig. 3(e) is thought to have a 
serious connection with the production of the Okhotsk Sea high. Fig. 3(f) shows the height distribution for $U=8 \mathrm{~m} / \mathrm{s}$, in which there is a weak ridge nearer to the mountain. As mentioned above, Fig. $3(\mathrm{e})$ is qualitatively similar to the normal height distribution in June (Fig. 4(b)), but there is, as in January, a discrepancy between the strengths of both general currents. But if we put $a=6,600 \mathrm{~km}$ instead of $a=8,000 \mathrm{~km}$ above, we can get pretty good values of the general current as may be seen from (24).

\section{The case when the general current is not uniform}

In Section 2 we assumed that general current $U$ was uniform. When $U$ is a function of $y$, it is extremely difficult to solve the equation (3) exactly. We will next deal with a linearized equation (12), that is

$$
\nabla^{2} z_{1}+\frac{\beta-U^{\prime \prime}}{U} z_{1}=-\lambda^{2} h
$$

In order to solve (26), we expand $-\lambda^{2} h, z_{1}$, and $\frac{\beta-U^{\prime \prime}}{U} z_{1}$ as follows :

$$
\begin{gathered}
-\lambda^{2} h=\sum_{i=1}^{n} F_{i}(x) \sin \frac{i \pi}{a} y, \\
z_{1}=\sum_{i=1}^{n} \phi_{i}(x) \sin \frac{i \pi}{a} y, \\
\frac{\beta-U^{\prime \prime}}{U} z_{1}=\sum_{i=1}^{n} A_{i}(x) \sin \frac{i \pi}{a} y .
\end{gathered}
$$

Substituting these into (26), we get the equations for $\phi_{i}(x)(i=1,2, \cdots \cdots, n)$ as follows :

$$
\frac{d^{2} \psi_{i}}{d x^{2}}-\left(\frac{i \pi}{a}\right)^{2} \phi_{i}+A_{i}(x)=F_{i}(x) . \quad(i=1,2, \cdots \cdots, n)
$$

On the other hand, from (28) and (29)

where

$$
\begin{aligned}
A_{i}(x) & =\frac{2}{a} \int_{0}^{a} z_{1}(x, \eta) \frac{\beta-U^{\prime \prime}(\eta)}{U(\eta)} \sin \frac{i \pi}{a} \eta d \eta \\
& =\frac{2}{a} \sum_{k=1}^{n} \phi_{k}(x) \int_{0}^{a} \frac{\beta-U^{\prime \prime}(\eta)}{U(\eta)} \sin \frac{i \pi}{a} \eta \sin \frac{k \pi}{a} \eta d \eta \\
& =\sum_{k=1}^{n} c_{i k} \phi_{k}(x), \quad(i=1,2, \cdots \cdots, n)
\end{aligned}
$$

$$
c_{i k}=\frac{2}{a} \int_{0}^{a} \frac{\beta-U^{\prime \prime}(\eta)}{U(\eta)} \sin \frac{i \pi}{a} \eta \sin \frac{k \pi}{a} \eta d \eta . \quad(i=1,2, \cdots \cdots, n)
$$

From (30) and (31), we may get

$$
\frac{d^{2} \psi_{i}}{d x^{2}}-\left(\frac{i \pi}{a}\right)^{2} \phi_{i}+\sum_{k=1}^{n} c_{i k} \psi_{k}=F_{i}(x) . \quad(i=1,2, \cdots \cdots, n)
$$

If we transform $\left\{\psi_{i}\right\}$ and $\left\{F_{i}\right\}$ as follows: 


$$
\begin{aligned}
\phi_{i}(x) & =\alpha_{i 1} \phi_{1}+\alpha_{i 2} \phi_{2}+\cdots \cdots+\alpha_{i n} \phi_{n}, \\
G_{i}(x) & =\alpha_{i 1} F_{1}+\alpha_{i 2} F_{2}+\cdots \cdots+\alpha_{i n} F_{n}, \quad(i=1,2, \cdots \cdots, n)
\end{aligned}
$$

then we can get

$$
\frac{d^{2} \phi_{i}}{d x^{2}}+\lambda_{i} \phi_{i}=G_{i}(x), \quad(i=1,2, \cdots \cdots, n)
$$

where $\lambda_{i}(i=1,2, \cdots \cdots, n)$ are $n$ roots of an algebraic equation

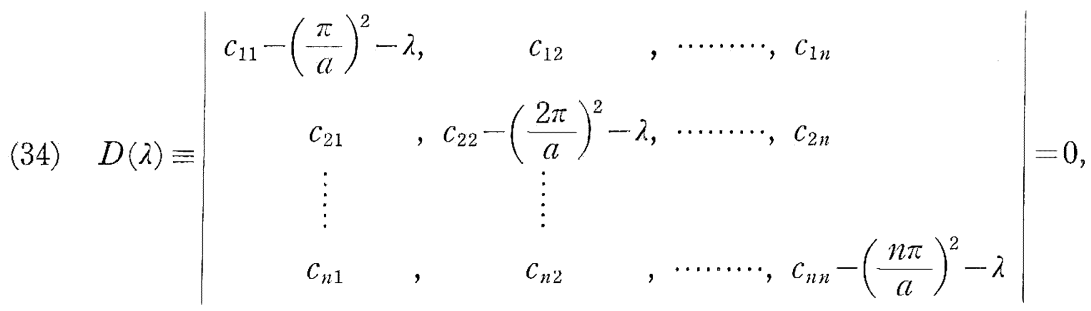

and $\alpha_{i 1}, \alpha_{i 2}, \cdots \cdots, \alpha_{i n}(i=1,2, \cdots \cdots, n)$ are $n$ sets of roots of the simultaneous equations

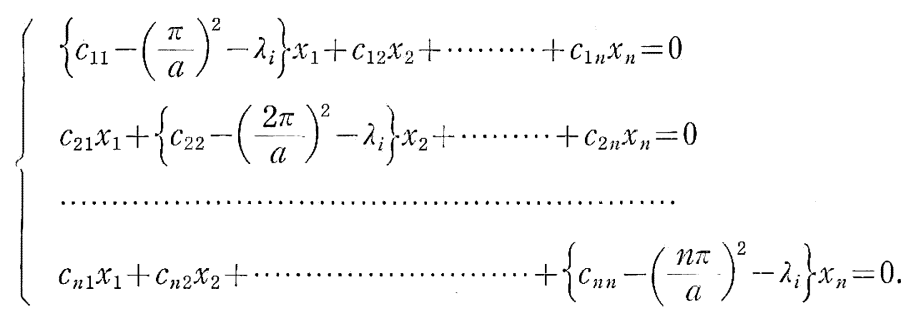

We will discuss the case when $U(y)$ is expressed as follows :

$$
U(y)=U_{0} e^{-\kappa\left(y-\frac{a}{2}\right)^{2}}
$$

where $\kappa=10^{-7} \mathrm{~km}^{-2}$ and $a=8,000 \mathrm{~km}$. For the sake of symmetry of $U(y), c_{12}=c_{14}$ $=c_{23}=c_{25}=c_{34}=c_{45}=0$, so that computing $c_{i k}$ of (31), (34) becomes as follows : $D\left(\lambda_{0}\right) \equiv$

$$
\begin{aligned}
& \begin{array}{llll}
1.28 \sigma_{0}-0.03-\lambda_{0}, \quad & 0 \quad 0.36 \sigma_{0}-0.1 \quad, \quad 0.12 \sigma_{0}-0.02
\end{array}
\end{aligned}
$$

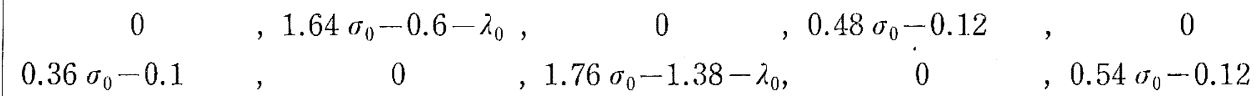

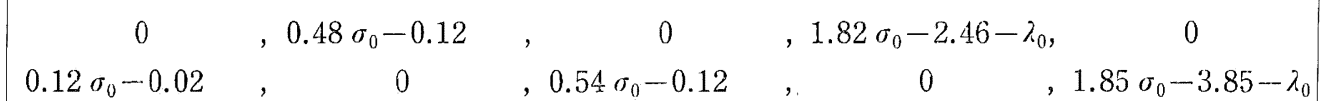

$$
\begin{aligned}
& =0 \text {, }
\end{aligned}
$$

where $\beta / U_{0}=\sigma_{0} \times 10^{-6} \mathrm{~km}^{-2}, \lambda=\lambda_{0} \times 10^{-6} \mathrm{~km}^{-2}$, and $n=5$. As may be seen from the form of the determinant $D(\lambda)$,

$$
D(\lambda)=D_{1}(\lambda) \cdot D_{2}(\lambda)
$$

where 


$$
\begin{aligned}
-D_{1}(\lambda) & =\left|\begin{array}{lll}
1.28 \sigma_{0}-0.03-\lambda, & 0.36 \sigma_{0}-0.1, & 0.12 \sigma_{0}-0.02 \\
0.36 \sigma_{0}-0.1, & 1.76 \sigma_{0}-1.38-\lambda, & 0.54 \sigma_{0}-0.12 \\
0.12 \sigma_{0}-0.02, & 0.54 \sigma_{0}-0.12, & 1.85 \sigma_{0}-3.85-\lambda
\end{array}\right| \\
D_{2}(\lambda) & =\left|\begin{array}{ll}
1.64 \sigma_{0}-0.6-\lambda, & 0.48 \sigma_{0}-0.12 \\
0.48 \sigma_{0}-0.12, & 1.82 \sigma_{0}-2.46-\lambda
\end{array}\right|
\end{aligned}
$$

Thus the algebraic equation $D(\lambda)=0$ is divided into the quadratic equation $D_{2}(\lambda)$ $=0$ and cubic equation $D_{1}(\lambda)=0$.

Therefore (32) becomes

$$
\begin{aligned}
& \frac{d^{2} \phi_{1}}{d x^{2}}-\left(\frac{\pi}{a}\right)^{2} \phi_{1}+c_{11} \psi_{1}+c_{13} \psi_{3}+c_{15} \psi_{5}=F_{1}(x), \\
& \frac{d^{2} \phi_{3}}{d x^{2}}-\left(\frac{3 \pi}{a}\right)^{2} \phi_{3}+c_{31} \phi_{1}+c_{33} \psi_{3}+c_{35} \psi_{5}=F_{3}(x), \\
& \frac{d^{2} \phi_{5}}{d x^{2}}-\left(\frac{5 \pi}{a}\right)^{2} \phi_{5}+c_{51} \phi_{1}+c_{53} \phi_{3}+c_{55} \psi_{5}=F_{5}(x), \\
& \frac{d^{2} \phi_{2}}{d x^{2}}-\left(\frac{2 \pi}{a}\right)^{2} \phi_{2}+c_{22} \psi_{2}+c_{24} \psi_{4}=F_{2}(x), \\
& \frac{d^{2} \phi_{4}}{d x^{2}}-\left(\frac{4 \pi}{a}\right)^{2} \phi_{4}+c_{42} \phi_{2}+c_{44} \phi_{4}=F_{4}(x),
\end{aligned}
$$

and (33) becomes

$$
\frac{d^{2} \phi_{i}}{d x^{2}}+\lambda_{i} \phi_{i}=G_{i}(x), \quad(i=1,2,3,4,5)
$$

where

$$
\left\{\begin{array}{rlrl}
\phi_{1} & =\alpha_{11} \phi_{1}+\alpha_{13} \phi_{3}+\alpha_{15} \psi_{5}, & & \dot{\phi}_{2}=\alpha_{22} \psi_{2}+\alpha_{24} \phi_{4}, \\
\phi_{3}=\alpha_{31} \phi_{1}+\alpha_{33} \phi_{3}+\alpha_{35} \psi_{5}, & & \phi_{4}=\alpha_{42} \psi_{2}+\alpha_{44} \phi_{4}, \\
\phi_{5}=\alpha_{51} \phi_{1}+\alpha_{53} \psi_{3}+\alpha_{55} \psi_{5} & \\
G_{1}=\alpha_{11} F_{1}+\alpha_{13} F_{3}+\alpha_{15} F_{5}, & G_{2}=\alpha_{22} F_{2}+\alpha_{24} F_{4}, \\
G_{3}=\alpha_{31} F_{1}+\alpha_{33} F_{3}+\alpha_{35} F_{5}, & G_{4}=\alpha_{42} F_{2}+\alpha_{44} F_{4}, \\
G_{5}=\alpha_{51} F_{1}+\alpha_{53} F_{3}+\alpha_{55} F_{5} . &
\end{array}\right.
$$

When $\lambda_{i}>0$, the solution of (33) is wave-shaped as in (20). Fig. 6 shows the wave-length of the above solution for varying $U_{0}$, maximum of the general current. This is similar to Fig. 1 when $U$ is regarded as an average value of the general current.

Next when $U_{0}=30 \mathrm{~m} / \mathrm{s}$, (37) becomes as follows :

$$
\begin{aligned}
& \phi_{1}=\phi_{1}+0.084 \phi_{3}+0.015 \phi_{5}, \\
& \phi_{3}=-0.085 \phi_{1}+\phi_{3}+0.068 \phi_{5}, \\
& \phi_{5}=-0.009 \phi_{1}-0.069 \phi_{3}+\phi_{5}, \\
& \phi_{2}=\phi_{2}+0.078 \phi_{4}, \\
& \phi_{4}=-0.078 \phi_{2}+\phi_{4} .
\end{aligned}
$$

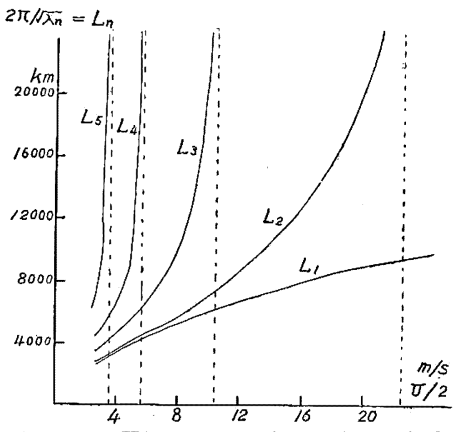

Fig. 6. The wave-lengths of he perturbations produced by the mountain when the general current was given by

$$
U=U_{0} e^{-\kappa\left(y-\frac{a}{2}\right)^{2}} .
$$


So that we may approximately put

$$
\phi_{i}=\phi_{i} . \quad(i=1,2,3,4,5)
$$

Thus the character of the flow pattern of this case is similar to the case when the general current is uniform.

\section{The effect of friction}

We will next discuss the effect of friction when the general current is uniform. When it is stationary, the linearized equation of (1) corresponding to (5) becomes as follows :

$$
\nabla^{2} \frac{\partial z_{1}}{\partial x}+\frac{\beta}{U} \cdot \frac{\partial z_{1}}{\partial x}+\frac{\kappa F}{U} \nabla^{2} z_{1}=-\lambda^{2} \frac{\partial h}{\partial x} .
$$

We expand $h$ and $z_{1}$ in the Fourier series as follows:

$$
\begin{aligned}
-\lambda^{2} h & =\sum_{n=1}^{\infty} F_{n}(x) \sin \frac{n \pi}{a} y, \\
z_{1} & =\sum_{n=1}^{\infty} \phi_{n}(x) \sin \frac{n \pi}{a} y .
\end{aligned}
$$

Substituting these in (38), we may get the equation for $\phi_{n}$ as follows :

$$
\frac{d^{3} \phi_{n}}{d x^{3}}+\left(\frac{\beta}{U}-\frac{n^{2} \pi^{2}}{a^{2}}\right) \frac{d \phi_{n}}{d x}+\frac{\kappa F}{U}\left(\frac{d^{2} \phi_{n}}{d x^{2}}-\frac{n^{2} \pi^{2}}{a^{2}} \phi_{n}\right)=\frac{d F_{n}}{d x} .
$$

In order to find the solution of equation (39), we must solve the algebraic equation

$$
X^{3}+\frac{\kappa F}{U} X^{2}+\left(\frac{\beta}{U}-\frac{n^{2} \pi^{2}}{a^{2}}\right) X-\frac{\kappa F n^{2} \pi^{2}}{U a^{2}}=0 .
$$

Fig. 7 shows the relations between the roots of $(40)$ and $U$, putting $n=1,2,3$, 4,5 when $F=4.0 \times 10^{-6} s^{-1}, \quad \kappa=0.4$ and $a=8,000 \mathrm{~km}$. In this figure, real roots are expressed by $r_{n}$, and imaginary roots by $\lambda_{n} \pm i \mu_{n}$. We may find the solutions of (39) corresponding to the above roots of (40) as follows, when the boundary conditions are

$$
\lim _{x \rightarrow-\infty} \phi_{n}=0, \quad \lim _{x \rightarrow \infty} \phi_{n}=\text { finite. }
$$

i) When the roots of (40) are $\lambda_{n}$ $\pm i \mu_{n}\left(\lambda_{n}<0\right)$ and $r_{n}>0$, the solution of (39) satisfying the boundary conditions becomes as follows:

$$
\text { (41) } \begin{aligned}
\phi_{n}(x)= & \frac{1}{\left(r_{n}-\lambda_{n}\right)^{2}+\mu_{n}^{2}} \\
& \times\left\{r_{n} \int_{\infty}^{x} F_{n}(\xi) e^{r_{n}(x-\xi)} d \xi\right.
\end{aligned}
$$

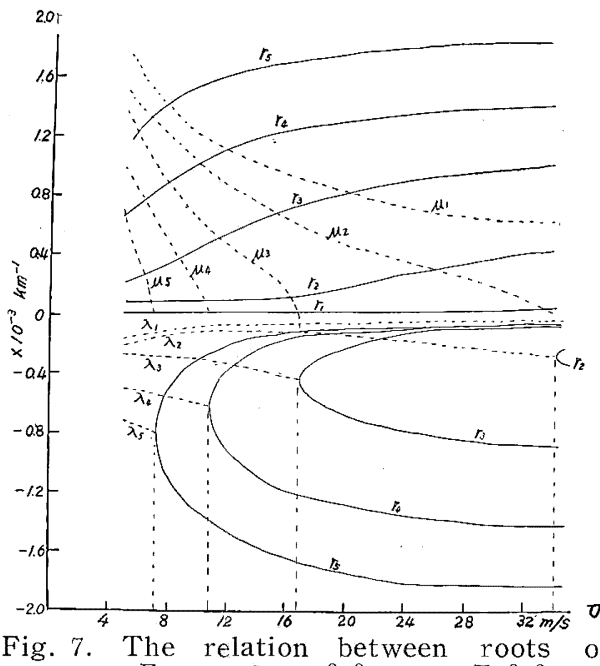
$x^{3}+\frac{\kappa F}{U} x^{2}+\left(\frac{\beta}{U}-\frac{n^{2} \pi^{2}}{a^{2}}\right) \times \frac{-\kappa F n^{2} \pi^{2}}{U a^{2}}=0$ and $U$, putting $n=1,2,3,4,5$ when $F=4 \times 1,0^{-6} s^{-1}, \kappa=0.4$ and $a=8,000 \mathrm{~km}$. Real roots are expressed by $r_{n}$ and imaginary roots by $\lambda_{n} \pm i u_{n}$. 


$$
\begin{aligned}
& -r_{n} \int_{-\infty}^{x} F_{n}(\xi) e^{\lambda_{n}(x-\xi)} \cos \mu_{n}(x-\xi) d \xi \\
& \left.+\frac{\lambda_{n}^{2}-\lambda_{n} r_{n}+\mu_{n}{ }^{2}}{\mu_{n}} \int_{-\infty}^{x} F_{n}(\xi) e^{\lambda_{n}(x-\xi)} \sin \mu_{n}(x-\xi) d \xi\right\} .
\end{aligned}
$$

ii) When the roots of (40) are all real and expressed by $r_{n}{ }^{(1)}(>0), r_{n}^{(2)}(<0)$, and $r_{n}{ }^{(3)}(<0)$, the solution of (39) is

$$
\begin{aligned}
& \left.\psi_{n}(x)=-\frac{r_{n}{ }^{(1)}}{\left(r_{n}{ }^{(1)}\right.}-r_{n}^{(2)}\right)\left(r_{n}^{(1)}-{\overline{r_{n}}}^{(3)}\right) \int_{+\infty}^{x} F_{n}(\xi) e^{r_{n}{ }^{(1)}(x-\xi)} d \xi \\
& +\frac{r_{n}{ }^{(2)}}{\left(r_{n}{ }^{(2)}-r_{n}{ }^{(1)}\right)\left(r_{n}{ }^{(2)}\right.}-\overline{\left.r_{n}{ }^{(3)}\right)} \int_{-\infty}^{x} F_{n}(\xi) e^{r_{n}{ }^{(2)}(x-\xi)} d \xi \\
& +\frac{r_{n}{ }^{(3)}}{\left(r_{n}{ }^{(3)}-r_{n}{ }^{(1)}\right)\left(r_{n}{ }^{(3)}-r_{n}{ }^{(2)}\right)} \int_{-\infty}^{x} F_{n}(\xi) e^{r_{n}{ }^{(3)}(x-\xi)} d \xi \text {. }
\end{aligned}
$$

iii) When the algebraic equation (40) has equal roots, we can get solution of (39) by putting $\iota_{n} \rightarrow 0$ in (41) as follows :

$$
\begin{aligned}
\phi_{n}(x)= & \frac{1}{\left(r_{n}-\lambda_{n}\right)^{2}}\left\{r_{n} \int_{\infty}^{x} F_{n}(\xi) e\right. \\
& +\lambda_{n}\left(\lambda_{n}-r_{n}\right) \int_{-\infty}^{x} F_{n}(\xi) \\
& \left.\times e^{\lambda_{n}(x-\xi)}(x-\xi) d \xi\right\} .
\end{aligned}
$$

The first term of (41) gives perturbation in the vicinity of the mountain and the second and third terms have the same character as (20) but also damping effect as going away from the mountain. The solution (43) is a critical case of (41) corresponding to (22). The solution (42) gives perturbations in the neighbourhood of the mountain. Fig. 8 shows the wave length $\frac{2 \pi}{\mu_{n}}$ of the damping wave for varying values of $U$.

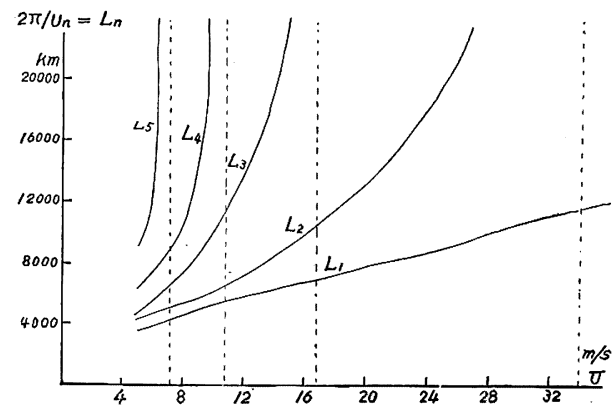

Fig. 8. The wave lengths of the perturbations produced by the mountain when the effect of friction was taken into account.

Acknowledgements - - The author wishes to express his thanks to Dr. H. Arakawa and Dr. S. Syono for their guidance and encouragement. He also wishes to thank Messrs. T. Murakami and Y. Masuda, and the staff members of N.W.P. group in Tokyo for their kind discussions.

\section{References}

Bomin, B., 1950: On the influence of the earth's orography on the general character of the westerlies. Tellus, 2, 148-195. 
Charney, J.G. and A. Eujassen, 1949: A numerical method for predicting the perturbations of the middle latitudes westerlies. Tellus, 1, 38-54.

Ganвo, K., 1956: The topographical effect upon the jet stream in the westerlies. Journ. Met. Soc. Japan, 34, 24-28.

Murakami, T., 1951: On the study of the change of the upper westerlies in the last stage of Baiu Season (Rainy Season in Japan). Journ. Met. Soc. Japan, 29, 162-175.

Murakami, T., 1956 : The to ographical effect upon the stationary upper flow patterns. Pap. Met. Geophys., 7, 69-89. 